\title{
Efecto del genotipo de Cavia porcellus en indicadores de reproducción y progenie, distrito Luya, Amazonas
}

\section{Effect of the genotype of Cavia porcellus on reproduction and progeny indicators, district Luya, Amazonas}

\author{
Jelber Tafur Sanchez ${ }^{1}$
}

\section{RESUMEN}

El objetivo de la investigación fue evaluar el efecto del genotipo de cuyes (Cavia porcellus) en indicadores de reproducción y progenie en el distrito de Luya, Amazonas. Se utilizaron 30 cuyes hembras y 05 cuyes machos reproductores de la línea sintética INIA, genotipo Cieneguilla UNALM, y genotipo Luya (Corporación Amazonas R\&G S.A.C.) haciendo un total de 105 cuyes reproductores $(90 \propto$ y $15 \AA$ ), distribuidos en tres tratamientos (T1: cuyes de la línea sintética, T2: cuyes genotipo Cieneguilla y T3: cuyes del genotipo luya) y cinco repeticiones por tratamiento. El experimento fue un diseño completamente al azar (DCA). Parámetros evaluados para el comportamiento reproductivo: tamaño de camada, tiempo empadre-parto, índice productivo, peso de reproductoras al inicio, peso de reproductores al destete y mortalidad; para el comportamiento productivo de la progenie se evaluó: peso nacimiento, peso primera semana, peso al destete y mortalidad. Las diferencias no resultaron significativas $(\mathrm{p}<0.05)$ entre los tratamientos, pero si se encontraron diferencias numéricamente donde los resultados mostraron que: los cuyes del genotipo Cieneguilla presentaron mayor tamaño de camada ( 2,80 crías por parto), menor tiempo de empadre-parto (76,53 días), mejor índice productivo (0.85), mayor número de crías destetadas $(2,57)$ y mejores pesos de reproductoras al destete $(1234.9 \mathrm{~g})$. En la evaluación de la progenie la línea sintética INIA presentó mejores valores: mayor peso al nacimiento $(133,80 \mathrm{~g})$ y mejor peso al destete $(267,03 \mathrm{~g})$. En conclusión, el genotipo Cieneguilla presentó mejores valores de reproducción y la línea sintética INIA como mejor en progenie.

Palabras clave: cuy, genética, productividad y progenie

\begin{abstract}
The objective of the research was to evaluate the effect of the genotype of guinea pigs (Cavia porcellus) on reproduction and progeny indicators in the district of Luya, Amazonas. 30 female guinea pigs and 05 breeding male guinea pigs of the synthetic line INIA, genotype Cieneguilla UNALM, and genotype Luya (Corporación Amazonas R\&G SAC) were used, making a total of 105 breeding guinea pigs (90 + and $15 \bigcirc^{\Uparrow}$ ), distributed in three treatments (T1 : guinea pigs of the synthetic line, T2: guinea pigs genotype Cieneguilla and T3: guinea pigs of the genotype luya) and five repetitions per treatment. The experiment was a completely randomized design (DCA). Parameters evaluated for reproductive behavior: litter size, mating-calving time, productive index, weight of breeders at the beginning, weight of breeders at weaning and mortality; for the productive behavior of the progeny, the following were evaluated: birth weight, first week weight, weaning weight and mortality. The differences were not significant $(\mathrm{p}<0.05)$ between the treatments, but numerical differences were found where the results showed that: the guinea pigs of the Cieneguilla genotype had greater litter size (2.80 young per parturition), shorter breeding time. calving (76.53 days), better productive index (0.85), greater number of weaned pups (2.57) and better weights of breeders at weaning $(1234.9 \mathrm{~g})$. In the evaluation of the progeny, the INIA synthetic line presented better values: higher birth weight $(133.80 \mathrm{~g})$ and better weaning weight $(267.03 \mathrm{~g})$. In conclusion, the Cieneguilla genotype presented better reproduction values and the INIA synthetic line had the best progeny.
\end{abstract}

Keywords: guinea pig, genetics, productivity and progeny

${ }^{1}$ Bachiller de la Facultad de Ingeniería Zootecnista, Agronegocios y Biotecnología. Universidad Nacional Toribio Rodríguez de Mendoza de Amazonas. Correo electrónico: 081018a112@untrm.edu.pe 


\section{INTRODUCCIÓN}

La crianza masiva de cuyes se extiende y exige la multiplicación de bases genéticas que le permiten acceder a una conservabilidad y rentabilidad a la crianza. En la actualidad, el país cuenta con tres poblaciones bien definidas, la mejorada constituido por razas puras y/o cruzamientos interraciales, las cruzadas y los ecotipos locales. El cruce interracial es una opción para producir variaciones genéticas en una población, puede conservarse el cruce entre razas puras (triple cruce) o conformar una línea sintética.

La conformación de la línea sintética consideró disponer una línea que acoplara las características individuales de cada raza (Perú, Andina e Inti) en un animal que tenga una productividad sostenible y rentable en los sistemas de producción masiva. La sumisión por forraje, como herbívoro que es el cuy, no permite el aumento poblacional en las granjas donde son empleadas como empresas grandes o pequeñas Chauca, et al (2015).

La línea sintética como todas las especies se ha conformado por el cruce de tres razas, sus índices productivos se vienen optimizando por medio de selección hasta constituir este biotipo. Se diferencia de las razas puras Perú, Andina e Inti en que es más versátil genéticamente porque está conformada por la suma del aporte de cada componente racial que ayudo en su procedencia. La mayor factibilidad, esta equiparado por sus niveles más altos de producción, debido a sus características genéticas superiores a la de sus padres.

La línea sintética P.625-14 tiene una mezcla de los caracteres deseables de las razas originales. Perú precoz, Andina prolífica e Inti precoz y prolífica con ello se buscó complacer la demanda de la producción y del mercado. Su conformación es una alternativa para aumentar la producción en las crianzas, familiar, familiar-comercial o comercial que utilicen bases genéticas puras que les permita producir sus propios cruzamientos. Para generar mayor impacto en la producción nacional, es indispensable emplear cuyes reproductores hembras regionales como vientres, de esta manera se introduce masiva y velozmente genes de alta productividad en la producción intensiva de cuyes a nivel del pequeño productor. Los índices productivos determinados de la línea sintética permiten imaginar que su utilización es válida para aumentar la producción de carne en las granjas comerciales. Chauca, et al.; (2014).

Los cuyes del genotipo Cieneguilla, desarrollado en la Universidad Nacional Agraria la Molina (UNALM), Perú, son el resultado de procesos de selección conducidos para lograr animales con mayor peso en un menor tiempo; sin embargo, existen muchas características de su desarrollo que no están bien definidas Rubio, et al.; (2019).
Los animales del genotipo Cieneguilla-UNALM, provienen del cruzamiento y consolidación de varios genotipos procedentes de La Molina (UNALM), Granja Santa María (Chorrillos-Lima), Granja de Animales Menores del INIA (La Molina- Lima), Granja de Yauris de la UNCP en el valle del Mantaro (Huancayo-Junín), y la Granja Auquicuy (IbarraEcuador); el genotipo formado se caracteriza por su rusticidad, velocidad de crecimiento de conformación corporal carnicera, robusta y redondeada Rubio, (2018).

Las características maternales del genotipo Cieneguilla; como total de nacidos, numero de nacidos vivos, numero de destetados, peso al nacimiento y peso al destete son variables de importancia económica para la producción de cuyes, lo cual deben ser introducidos en los programas de mejoramiento genético, el total de nacidos es una característica maternal que están influidos por factores como peso de la madre al parto, numero de parto, efecto genético y sistema de apareamiento. La variable, numero de cuyes destetados está influenciada por factores como: habilidad materna, número de parto de la madre, tamaño de camada al nacimiento y mortalidad de las crías del nacimiento al destete Rodriguez, et al.; (2015)

La finalidad de la presente investigación fue evaluar el efecto del genotipo de cuyes (Cavia porcellus) en indicadores de reproducción y progenie en el distrito de Luya, región Amazonas, Perú.

\section{MATERIAL Y MÉTODO}

\section{Lugar de ejecución}

El área experimental se desarrolló en la región Amazonas, Provincia de Luya, distrito de Luya en el sector el Oratorio, a una altitud de $2316 \mathrm{msnm}$ y coordenadas UTM: 18 M 174995 E y 9317952 S.

\section{Material Experimental}

- Cuyes para reproductoras de 30 días hembras y 45 de días machos de la línea sintética INIA, genotipo Cieneguilla UNALM y genotipo Luya (Corporación Amazonas R\&G S.A.C.).

\section{Actividades a realizar}

Se construyeron jaulas de $0.9 \mathrm{~m} \times 1.50 \mathrm{~m} \times 1.05 \mathrm{~m}$ (A, $\mathrm{L}, \mathrm{H})$ para alojamiento de reproductoras durante la etapa de empadre a fin de mantener su zona de confort.

Los cuyes del distrito de Luya fueron considerados del núcleo genético que tiene actualmente la empresa donde se desarrolló el trabajo de investigación, así mismo, las reproductoras del genotipo Cieneguilla se transportaron desde la Universidad Nacional Agraria la Molina - Lima hasta el centro de investigación, al igual que la línea sintética INIA-Lima. 
Después de la adquisición de los reproductores, se colocaron en una densidad de 6 hembras por jaula y 05 machos por jaula para el proceso de adaptación, hasta que alcancen la edad y peso de empadre.

La alimentación de reproductoras fue mixta basado en alfalfa (leguminosa), maralfalfa (Gramínea) y concentrado (18\% PT y $28 \mathrm{Mcal})$. El reparto de alimento se suministró considerando su consumo en base a materia seca (con relación a su peso vivo $5 \%$ ), proporcionándoles agua ad libitum mediante red de tubería con chupones automatizados, el horario del suministró de alimento fue a las 8:00 am y a las 4:30 pm dos raciones diarias.

Para la distribución de tratamientos se inició con el aretado de las reproductoras y del reproductor, sucesivamente se procedió a distribuir en módulos, donde se alojaron 06 hembras más 01 macho los cuales conformaron una unidad experimental desarrollados durante la etapa de empadre y la ubicación de cada tratamiento fue de manera aleatoria, uniforme y con repetibilidad.

\section{Tratamientos}

\begin{tabular}{|c|c|c|c|c|}
\hline Rep. & Trat. & $\begin{array}{l}\mathrm{T}_{1} \\
\mathrm{a}_{1}\end{array}$ & $\begin{array}{l}\mathrm{T}_{2} \\
\mathrm{a}_{2}\end{array}$ & $\begin{array}{l}\mathrm{T}_{3} \\
\mathrm{a}_{3}\end{array}$ \\
\hline 1 & & $\mathrm{r}_{1}$ & $\mathrm{r}_{1}$ & $r_{1}$ \\
\hline 2 & & $\mathrm{r}_{2}$ & $r_{2}$ & $r_{2}$ \\
\hline 3 & & $r_{3}$ & $r_{3}$ & $r_{3}$ \\
\hline 4 & & $\mathrm{r}_{4}$ & $r_{4}$ & $r_{4}$ \\
\hline 5 & & $r_{5}$ & $r_{5}$ & $r_{5}$ \\
\hline
\end{tabular}

Donde:

$\mathrm{a}_{1}=$ Cuyes de la línea sintética INIA

$\mathrm{a}_{2}=$ Cuyes del genotipo Cieneguilla UNALM

$\mathrm{a}_{3}=$ Cuyes del genotipo Luya

$\mathrm{r}=$ repeticiones

\section{Variables de estudio}

- Variables independientes

$\mathrm{a}_{1}=$ Cuyes de la línea sintética INIA

$\mathrm{a}_{2}=$ Cuyes Genotipo Cieneguilla UNALM

$\mathrm{a}_{3}=$ Cuyes genotipo Luya (Corporación Amazonas R\&G S.A.C.)

- Variables dependientes

$\mathrm{y}_{1}=$ Comportamiento reproductivo

$\mathrm{y}_{1.1}=$ Tamaño de camada (1 er parto)

$\mathrm{y}_{1.2}=$ Tiempo empadre - parto

$\mathrm{y}_{1.3}=$ Índice productivo IP

$\mathrm{y}_{1.4}=$ Peso de reproductoras (inicio y destete)

$\mathrm{y}_{1.5}=$ Mortalidad en reproductoras

$\mathrm{y}_{2}=$ Producción de la progenie

$\mathrm{y}_{21}=$ Pesos (nacimiento, primera semana $\mathrm{y}$ destete)

$\mathrm{y}_{2.2}=\%$ mortalidad (aborto, nacimiento y lactancia)

Finalmente: se trabajó con 3 tratamientos y 5 repeticiones $=3 \times 5=15$ unidades experimentales y 1 $\mathrm{UE}=1$ módulo (6 hembras y 1 macho).

Finalmente: se trabajó con 3 tratamientos y 5 repeticiones $=3 \times 5=15$ unidades experimentales y 1 $\mathrm{UE}=1$ módulo ( 6 hembras y 1 macho).

\section{Diseño experimental}

En la presente investigación se utilizó un diseño completamente al azar (DCA), con 3 tratamientos y 5 repeticiones, Se llevó a cabo el análisis de variancia para establecer las diferencias significativas entre los tratamientos y la prueba de Tuckey para confrontar las medias de los tratamientos en los parámetros estimados. Se trabajó con un nivel de significancia de 0.05 .

Modelo aditivo lineal: Es un modelo aditivo lineal Tipo 1:

$$
\mathbf{Y i j}=\boldsymbol{\mu}+\mathbf{t i}+\varepsilon \mathbf{i j}
$$

Donde:

$\mathrm{Y}_{\mathrm{ij}} \quad=$ Observación experimental.

$\mu \quad=$ Efecto de la media general.

ti $=$ Efecto del i-ésimo tratamientos

$\varepsilon_{\mathrm{ij}}=$ Error experimental

Los resultados alcanzados se sometieron a un análisis de varianza ANVA para determinar las diferencias significativas o no entre tratamiento. Nivel de significación $(\propto)=5 \%$ y nivel de confianza $(1-\alpha)=$ $95 \%$.

\section{RESULTADOS}

Tabla 3

Tamaño de camada (1er parto)

\begin{tabular}{lc}
\hline Genotipo de cuyes & $\begin{array}{l}\text { Tamaño de camada } \\
(1 \text { er parto) }\end{array}$ \\
\hline Línea Sintética INIA & $2.57 \pm 0.22 \mathrm{a}$ \\
Cieneguilla UNALM & $2.80 \pm 0.38 \mathrm{a}$ \\
Luya & $2.40 \pm 0.22 \mathrm{a}$ \\
\hline a,b Letras diferentes en cada fila indican diferenc \\
significativas $(\mathrm{p}<0.05)$
\end{tabular}

En la tabla 3 los resultados muestran para la variable tamaño de camada en el primer parto no existió diferencias significativas $(\mathrm{p}=0.127)$, sin embargo, se observa que numéricamente los cuyes del genotipo Cieneguilla presentaron mayor tamaño de camada con 2.80 crías/parto superando a la línea sintética INIA y genotipo Luya con (2.57 y 2.40 respectivamente). 
Tabla 4

Tiempo empadre - parto (días)

\begin{tabular}{lc}
\hline Genotipo de Cuyes & $\begin{array}{c}\text { Tiempo empadrparto } \\
\text { (días) }\end{array}$ \\
\hline Línea Sintética IN I $/$ & $76.83 \pm 6.25 \mathrm{a}$ \\
Cieneguilla UNA L & $76.53 \pm 4.07 \mathrm{a}$ \\
Luya & $76.57 \pm 7.72 \mathrm{a}$ \\
\hline a,b Letras diferentes en cada fila indican difere \\
significativas $(\mathrm{p}<0.05)$
\end{tabular}

En la tabla 4 observamos que para la variable empadre-parto no existen diferencias significativas $(p=0.0996)$, sin embargo, se observa que numéricamente el genotipo Cieneguilla presenta menor tiempo de empadre con 76.53 días en comparación al genotipo Luya y línea sintética INIA con $(76.57$ y 76.83 respectivamente).

Tabla 5

Índice productivo

\begin{tabular}{lc}
\hline Genotipo de Cuyes & IP. \\
\hline Línea Sintética INIA & 0.76 \\
Cieneguilla UNALM & 0.84 \\
Luya & 0.72 \\
\hline
\end{tabular}

En la tabla 5 Se observa el índice productivo de los tratamientos evaluados en donde se determina el número de cuyes logrados por madre por mes, encontrando que el genotipo Cieneguilla UNALM presenta un valor de 0.84 superando a la línea sintética INIA y genotipo Luya $(0.76$ y 0.72 respectivamente), esto en términos numéricos.

Tabla 6

Números de crías destetadas

\begin{tabular}{lc}
\hline Genotipo de Cuyes & $\mathbf{N}^{\circ}$ de crías destetadas \\
\hline Línea Sintética INIA & $2.33 \pm 0.29 \mathrm{a}$ \\
Cieneguilla UNALM & $2.57 \pm 0.52 \mathrm{a}$ \\
Luya & $2.20 \pm 0.22 \mathrm{a}$ \\
\hline
\end{tabular}

${ }^{\mathrm{a}, \mathrm{b}}$ Letras diferentes en cada fila indican diferencias significativas $(\mathrm{p}<0.05)$

En la tabla 6 para la variable número de crías destetadas no se encontraron diferencias significativas $(\mathrm{p}=0.313)$, pero analizando numéricamente el genotipo Cieneguilla presenta mejores resultados con 2.57 crías destetadas.
Tabla 7

Peso de reproductoras (inicio)

\begin{tabular}{lc}
\hline Genotipo de Cuyes & $\begin{array}{c}\text { Peso de reproductoras g. } \\
\text { (inicio) }\end{array}$ \\
\hline Línea Sintética INIA & $884.47 \pm 9.48 \mathrm{a}$ \\
Cieneguilla UNALM & $884.00 \pm 7.18 \mathrm{a}$ \\
Luya & $879.80 \pm 10.53 \mathrm{a}$ \\
$\mathrm{a}, \mathrm{b}$ Letras diferentes en cada fila indican diferencias \\
significativas $(\mathrm{p}<0.05)$
\end{tabular}

En la tabla 7 observamos que la línea sintética INIA y genotipo Cieneguilla presenta mejores valores al momento de empadre o inicio con $884.47 \mathrm{~g}$ y $884.00 \mathrm{~g}$ respectivamente y no se encontraron diferencias significativas $(p=0.723)$.

\section{Tabla 8}

Peso de reproductoras (destete)

\begin{tabular}{lc}
\hline Genotipo de Cuyes & $\begin{array}{c}\text { Peso de reproductoras } \\
\text { g. (destete) }\end{array}$ \\
\hline Línea Sintética INIA & $1223.63 \pm 199.39 \mathrm{a}$ \\
Cieneguilla UNALM & $1234.90 \pm 208.02 \mathrm{a}$ \\
Luya & $1145.70 \pm 123.85 \mathrm{a}$ \\
\hline
\end{tabular}

${ }^{a, b}$ Letras diferentes en cada fila indican diferencias significativas $(\mathrm{p}<0.05)$

En la tabla 8 se observa que el genotipo Cieneguilla presenta mejores resultados en peso al destete con $1234.90 \mathrm{~g}$, seguido de la línea sintética INIA con $1223.63 \mathrm{~g}$ y finalmente el genotipo Luya con $1145.70 \mathrm{~g}$ y no se encontraron diferencias significativas $(\mathrm{p}=0.934)$.

\section{Tabla 9}

Ganancia de peso de reproductoras destete - inicio

\begin{tabular}{lc}
\hline Genotipo de Cuyes & Ganancia de peso \\
\hline Línea Sintética INIA & $339.2 \pm 192.3 \mathrm{a}$ \\
Cieneguilla UNALM & $350.9 \pm 210.4 \mathrm{a}$ \\
Luya & $265.9 \pm 123.7 \mathrm{a}$ \\
\hline a,b Letras diferentes en cada fila indican \\
diferencias significativas $(\mathrm{p}<0.05)$
\end{tabular}

En la tabla 9 los resultados mostraron que la ganancia de peso al destete con respecto a la etapa de inicio, no existió diferencias significativas $(\mathrm{p}=0.726)$, por lo cual se puede asumir que los cuyes presentaron las mismas ganancias de peso. 
Tabla 10

Mortalidad en reproductoras

\begin{tabular}{lc}
\hline Genotipo de Cuye & $\begin{array}{c}\text { Mortalidaden } \\
\text { reproductoras }\end{array}$ \\
\hline Línea Sintética IN ] & $0.03 \pm 0.07$ \\
Cieneguilla UNA L & $0.03 \pm 0.07$ \\
Luya & $0.03 \pm 0.07$ \\
\hline a,b Letras diferentes en cada fila indican
\end{tabular}

diferencias significativas $(p<0.05)$

En la tabla 10 se observa claramente que la mortalidad en las reproductoras fueron el mismo valor para los tres genotipos.

\section{Tabla 11}

Peso al nacimiento según genotipo de cuyes

\begin{tabular}{lc}
\hline Genotipo de Cuyes & Peso (nacimiento) \\
\hline Línea Sintética INIA & $133.80 \pm 27.44 \mathrm{a}$ \\
Cieneguilla UNALM & $128.27 \pm 21.60 \mathrm{a}$ \\
Luya & $111.00 \pm 10.75 \mathrm{a}$ \\
\hline a,b Letras diferentes en cada fila indican \\
diferencias significativas $(\mathrm{p}<0.05)$
\end{tabular}

En la tabla 11 observamos que la progenie de la Línea Sintética INIA presentaron mejores valores en peso al nacimiento con $133.80 \mathrm{~g}$, superando al genotipo Cieneguilla y genotipo luya con (128.27 y $111.00 \mathrm{~g}$ respectivamente), esto en términos numéricos mas no se encontró diferencias significativas $(\mathrm{p}=0.524)$.

\section{Tabla 12}

Peso a la semana 1según genotipo de cuyes

\begin{tabular}{lc}
\hline Genotipo de Cuyes & Peso (semana 1) \\
\hline Línea Sintética INIA & $199.27 \pm 37.38 \mathrm{a}$ \\
Cieneguilla UNALM & $195.13 \pm 31.79 \mathrm{a}$ \\
Luya & $175.93 \pm 17.15 \mathrm{a}$ \\
\hline a,b Letras diferentes en cada fila indican diferencias \\
significativas $(\mathrm{p}<0.05)$
\end{tabular}

En la tabla 12 observamos que la línea sintética INIA presenta mejor valor en peso al a semana 1 con $199.27 \mathrm{~g}$, seguido por el genotipo Cieneguilla con $195.13 \mathrm{~g}$ y finalmente el genotipo Luya con $175.93 \mathrm{~g}$ numéricamente y no se encontraron diferencias significativas $(p=0.725)$. Genotipo de Cuyes Ganancia de peso Línea Sintética INIA65.47 \pm 10.01 aCieneguilla UNALM66.87 \pm 12.59 aLuya64.93 \pm $6.61 \mathrm{a}$
Tabla 13

Ganancia de peso semana 1 - Peso nacimiento

\begin{tabular}{lc}
\hline Genotipo de Cuyes & Ganancia de peso \\
\hline Línea Sintética INIA & $65.47 \pm 10.01 \mathrm{a}$ \\
Cieneguilla UNALM & $66.87 \pm 12.59 \mathrm{a}$ \\
Luya & $64.93 \pm 6.61 \mathrm{a}$ \\
\hline
\end{tabular}

${ }^{a, b}$ Letras distintas en cada fila denotan diferencias significativas $(\mathrm{p}<0.05)$

En la tabla 13 los resultados mostraron que la ganancia de peso de la progenie en la semana 1 con respecto al peso de nacimiento, no existió diferencias significativas $(\mathrm{p}=0.952)$, asumiendo que los cuyes presentaron similares ganancias de peso para los tres genotipos, esto en términos numéricos.

\section{Tabla 14}

Peso al destete según genotipo de cuyes

\begin{tabular}{lc}
\hline Genotipo de Cuyes & Peso (destete) \\
\hline Línea Sintética INIA & $267.03 \pm 48.91 \mathrm{a}$ \\
Cieneguilla UNALM & $260.10 \pm 41.22 \mathrm{a}$ \\
Luya & $240.60 \pm 23.47 \mathrm{a}$ \\
\hline a,b Letras diferentes en cada fila indican \\
diferencias significativas $(\mathrm{p}<0.05)$
\end{tabular}

En la tabla 14 la línea sintética INIA presentó mejores valores numéricos en peso al destete con $267.03 \mathrm{~g}$ a diferencia de los genotipos Cieneguilla UNALM y Luya con 260.10 y $240.60 \mathrm{~g}$ respectivamente y no se encontraron diferencias significativas $(p=0.625)$.

\section{Tabla 15}

Ganancia de peso destete - Peso nacimiento

\begin{tabular}{lc}
\hline Genotipo de Cuyes & Ganancia de peso \\
\hline Línea Sintética INIA & $133.23 \pm 21.55 \mathrm{a}$ \\
Cieneguilla UNALM & $131.83 \pm 20.27 \mathrm{a}$ \\
Luya & $129.60 \pm 12.99 \mathrm{a}$ \\
\hline $\mathrm{a}, \mathrm{b}$ & Letras distintas en cada fila denotan diferencias
\end{tabular}
significativas $(\mathrm{p}<0.05)$

En la tabla 15 los resultados mostraron que la ganancia de peso de la progenie al destete con respecto al nacimiento, no existió diferencias significativas $(\mathrm{p}=0.953)$, por el cual se puede asumir que los cuyes presentaron similares ganancias de peso para los tres genotipos, en términos numéricos. 
Tabla 16

Mortalidad (Aborto)

\begin{tabular}{lc}
\hline Genotipo de Cuyes & Mortalidad (Aborto) \\
\hline Línea Sintética INIA & $0.03 \pm 0.07$ \\
Cieneguilla UNALM & 0 \\
Luya & 0 \\
\hline
\end{tabular}

En la tabla 16 la línea sintética INIA presentó abortos durante la investigación

Tabla 17

Mortalidad al nacimiento

\begin{tabular}{lc}
\hline Genotipo de Cuyes & $\begin{array}{c}\text { Mortalidad } \\
\text { (nacimiento) }\end{array}$ \\
\hline Línea Sintética INIA & $0.07 \pm 0.09$ \\
Cieneguilla UNALM & $0.10 \pm 0.15$ \\
Luya & $0.07 \pm 0.15$ \\
\hline
\end{tabular}

En la tabla 17 observamos que el genotipo Cieneguilla presento la mayor tasa de mortalidad en la etapa de nacimiento (0.10) seguido por la Línea Sintética INIA y genotipo Luya quienes presentaron valores inferiores 0.07 .

Tabla 18

Mortalidad en lactancia

\begin{tabular}{lc}
\hline Genotipo de Cuyes & Mortalidad (lactancia) \\
\hline Línea Sintética INIA & $0.13 \pm 0.14$ \\
Cieneguilla UNALM & $0.13 \pm 0.07$ \\
Luya & $0.17 \pm 0.12$ \\
\hline
\end{tabular}

En la tabla 18 observamos que la mortalidad en la etapa de lactación es mayor en el genotipo Luya (0.17) seguidos de la Línea Sintética INIA y genotipo Cieneguilla UNALM con 0.13 .

\section{DISCUSIÓN}

Respecto a la variable peso al nacimiento y destete, los resultados de la presente investigación se muestran dentro del rango de los resultados obtenidos por (Rubio, et al., 2019), quien evaluó el peso al nacimiento y destete de cuyes del genotipo Cieneguilla según el sexo de la cría, obteniendo como resultados que las hembras con peso al nacimiento y al destete fueron de 144.87 y $277.21 \mathrm{~g}$ respectivamente, por otro lado, en machos obtuvieron valores de 144.87 y $278.49 \mathrm{~g}$ respectivamente y sin presentar diferencia significativa $(P>0,05)$ entre hembras y machos. Los pesos al nacimiento observados en la presente investigación fueron similares, donde en el genotipo Cieneguilla obtuvo mejores pesos al nacimiento y al destete (128.27 y $260.10 \mathrm{~g}$ respectivamente), no encontrando diferencias significativas $(\mathrm{p}>0.05)$.

Al realizar el peso de reproductoras al destete, en la presente investigación se obtuvo resultados similares al rango obtenido por (Chauca, et al., 2013), quien evaluo el peso de reproductoras al parto y al destete 14 dias (1378.19 y $1293.58 \mathrm{~g}$ respectivamente); mientras que en el distrito de Luya se obtuvo $1233.44 \mathrm{~g}$ en cuanto al peso de reproductoras al destete de la Linea sintetica

INIA, no encontrando diferencias significativas $(p>0.05)$.

El tamaño de camada al primer parto de la línea sintética INIA, arrojó resultados superiores ( $2.57 \pm 0.22$ crías por parto) respecto a la investigación realizada por (Chauca, et al., 2017) quienes obtubieron resultados de 2.22 crias por parto de la raza Perú con respecto al tamaño de camada; asi mismo para el genotipo Cieneguilla con respecto a la variable tamaño de camada al primer parto se obtuvo resultados de $2.80 \pm 0.38$ crías por parto, siendo similares a los resultados generados por (Rodriguez, et al., 2015), quienes tuvieron resultados de $2.92 \pm 0.2$ crías por parto.

El peso al destete es una de las variables muy importantes en la producción de cuyes, por ello (Rodriguez, et al., 2013) en su investigación evaluó el peso al destete, teniendo como resultado un peso promedio (310.9 $\pm 96.3 \mathrm{~g})$ donde la edad promedio fue a los 14.2 días. Sin embargo, en esta investigación se obtuvieron resultados similares para la variable peso al destete para el genotipo Cieneguilla UNALM y línea sintética INIA logrando un peso promedio de (260.10 \pm 41.22 y $267.03 \pm 48.91 \mathrm{~g}$ respectivamente), no encontrando diferencias significativas $(\mathrm{p}>0.05)$.

El cuy mejorado tiene un promedio de número de crías por parto de 2.8 a 3.9. Presentan mayor frecuencia en cuanto a la aparición de celos después del parto, llega a los $800 \mathrm{~g}$ de peso a los 3.2 meses de edad (Balarezo, 2017). Sin embargo, el genotipo Cieneguilla UNALAM presentó menor tiempo de empadre con 76.53 días. Por otro lado, tanto la línea sintética INIA como el genotipo Cieneguilla presentaron mejores valores en cuanto al peso de reproductoras al inicio con $884.47 \mathrm{~g}$ y $884.00 \mathrm{~g}$ respectivamente, pero cabe resaltar que el genotipo Cieneguilla presento mejores resultados en cuanto al peso de reproductoras al destete con $1234.9 \mathrm{~g}$ a pesar que no se encontraron diferencias significativas $(\mathrm{p} \geq 0.005)$.

\section{CONCLUSIONES}

Los cuyes reproductores del genotipo Cieneguilla mostraron tamaño de camada superior ( 2.80 crías por parto) a la línea sintética INIA y genotipo Luya (2.57 y 2.44 crías por parto respectivamente), valores superiores respecto al número de crías destetadas (2.57 crías por camada), menor tiempo empadreparto (76.53 \pm 4.07 días) respecto al genotipo luya y genotipo Cieneguilla ( $76.57 \pm 7.72$ y $76.83 \pm 6.25$ días respectivamente), del mismo modo tanto la línea 
sintética como el genotipo Cieneguilla presentaron mejores resultados durante peso de reproductoras al inicio $(884.47 \pm 9.48$ y $884.00 \pm 7.18 \mathrm{~g}$ respectivamente), pero cabe resaltar que el genotipo Cieneguilla mostro mejores resultados en cuanto al peso de reproductoras al destete $(1200 \mathrm{~g})$ esto en términos numéricos mas no se encontró diferencia significativa ( $\mathrm{p} \geq 0.05)$ debido a que los cuyes fueron sometidos a un mismo sistema tales como: igual alimentación, ambiente, manteniendo siempre el principio de control local, uniformidad y repetitividad.

En cuanto a la evaluación de la progenie, la línea sintética INIA mostro valores superiores en peso al nacimiento, peso a la primera semana y peso al destete $(133.80 \pm 27.44,199.27 \pm 37.38$ y 267.03 $\pm 48.91 \mathrm{~g}$ respectivamente) al genotipo Cieneguilla UNALM y genotipo Luya $(128.27 \pm 21.60,195.13 \pm$ $31.79,260.10 \pm 41.42 \mathrm{~g}$ y $111.00 \pm 10.75,175.93 \pm$ 17.15 y $240.60 \pm 41.22 \mathrm{~g}$ respectivamente) esto en términos numéricos mas no se encontró diferencia significativa $(\mathrm{p} \geq 0.05)$ debido a la uniformidad del trabajo que se realizó ya que todos los tratamientos partieron bajos las mismas condiciones.

\section{REFERENCIAS BIBLIOGRÁFICAS}

Balarezo, F. (2017). Evaluación Reproductiva de tres Lineas de Cavia porcellus (cuy) Perú, Andina e Inti Criados en Jaulas en condiciones de Selva Alta-Satipo. Tesis, Universidad Nacional del Centro del Perú, satipo-Perú.

Chauca, L.; Huaman, M. y Remigio, I. (2014). Parámetros productivos de una línea interracial de cuyes Cavia porcellus sintetica P.625-14. Crianzas Menores: EL CUY, 1-9.

Chauca, L.; Huaman, M.; Reynaga, M.; Muscari, J. y Higaonna, R. (4-6 de Diciembre de 2013). Comportamiento Reproductivo de Cuyes de Linea Sintética (INIAP 5/8 IxA3/8). 1-3.

Chauca, L.; Muscari, J.; Higaonna, O. y Huaman, M. (2017). Evaluacion de la Vida Productiva de Cuyes Cavia porcellus de una Linea Sintética P 0.63-310 manejada en la Costa Central. Agro Enfoque, 1-8.

Chauca, L.; Valencia, R. y Muscari, J. (2015). Crecimiento de dos líneas de cuyes (Cavia porcellus) con dos sistemas de alimentación. Crianza de Animales Menores: EL CUY, 1-5.

Macancela, M. (2019). Evaluación de parámetros zootécnicos a distintas edades del destete en engorde de cobayos (Cavia porcellus). Tesis, Universidad Politécnica Salesiana Sede
Cuenca, Cuenca - Ecuador.

Rodriguez, H.; Gutiérrez, G.; Palomino, M. y Hidalgo, V. (Enero-Marzo de 2015). Características Maternales al Nacimiento y Destete en cuyes de Costa Central del Perú. Revista de investigaciones Veterinarias del Perú, RIVEP, 26(1), 77-85.

Rodriguez, H.; Palomino, M.; Hidalgo, V. y Gutierrez, G. (2013). Efectos de Factores Fijos y al Azar Sobre el Peso al Nacimiento y al Destete en cuyes de la Costa Central del Perú. Rev Inv Vet Perú, 24(1), 16-24.

Rubio, P. (2018). Estimación de Parámetros Fenotípicos y Genéticos para medida de Carcasa en Cuyes (Cavia porcellus) del Genotipo Cieneguilla. Lima-Perú: Universidad Nacional Agraria la Molina.

Rubio, R.; Deza, H.; Alvarado, J.; Castillo, E. y Maldonado, M. (Enero-Febrero de 2019). Efecto del Sexo, Tamaño de camado y numero de parto sobre los pesos al nacimiento y al destete de cobayos (Cavia porcellus) del genotipo cieneguilla. Serbiluz, XXIX (1), 1-8. 